MODELING, IDENTIFICATION AND CONTROL, 2000, VOL. 21, NO. 2, 83-103

doi:10.4173/mic.2000.2.2

\title{
Evaluation of Dynamic Models of Distillation Columns with Emphasis on the Initial Response
}

\author{
BERND WITTGENS and SIGURD SKOGESTAD*†
}

Keywords: Distillation dynamics, tray hydraulics, experimental response

The flow dynamics (tray hydraulics) are of key importance for the initial dynamic response of distillation columns. The most important parameters are the liquid holdup, the liquid hydraulic time constant and the vapor constant representing the initial effect of a change in vapor flow on liquid flow. In the paper we present methods for determining these parameters experimentally, and compare the results with estimates from available correlations such as the Francis Weir formula.

\section{Introduction}

The objective when deriving a model is to make it as simple as possible while at the same time matching the dynamics of the real system. The estimation of the dynamic parameters which determine the behavior of the process is of crucial importance in the modeling process. For control purposes the most important feature of a model is to match the dynamic response at times corresponding to the desired closed-loop time constant. This means that if the dynamic model is to be used for evaluating and tuning the 'fast' loops on a distillation column (pressure loop, level loops, temperature profile 'stabilization') where the time constants are in the order of a few minutes or less, then a good model for the initial response is required and accurate steady state behavior may be less important.

Simplified models which simplify or neglect flow dynamics (tray hydraulics) and energy balance are often used for studies of distillation column dynamics and control. However, the applicability of such simple models for this purpose is often questioned by practitioners. This critique is indeed reasonable as one knows that the tray hydraulics are crucial in determining the initial dynamic response which is of key importance for control. Although the essential dynamics of a distillation column can be obtained from simplified models, the introduction of realistic and accurate hydraulic calculations allows us to study the operability of a given system and the design and evaluation of complex control systems.

In the paper we consider detailed models of the tray hydraulics and use these to derive expressions for parameters that characterize the flow response. The most important parameters are the liquid holdup $M_{l}$, the hydraulic time constant $\tau_{l}$, the parameter $\lambda$ (denoted $K_{2}$ by many authors) for the initial effect of a change in vapor flow on liquid flow, the fraction of vapor on the tray and the pressure drop. These key parameters are also determined from experiments on our lab-scale column. The final goal of these studies is to see how detailed a dynamic model of a distillation column should be in order to be used for control purposes.

\footnotetext{
Presented at: IFAC-symposium DYCORD + '95, 7-9 June 1995, Helsingør, Denmark.

* Norwegian University of Science and Technology (NTNU), Department of Chemical Engineering, 7491 Trondheim, Norway.

$\dagger$ Author to whom correspondence should be addressed, phone: +47 7359 4154, fax: +47 7359 4080, E-mail: skoge@chembio.ntnu.no.
} 
The literature on distillation dynamics is extensive so only a short overview will be given. In terms of experimental responses for tray columns we only mention the work of Baber et al. $(1961,1962)$. Open- and closed-loop experiments under automatic control were performed, additionally a linearized deviation model were presented and compared to the recorded transient responses. Perforated plates have been used extensively for liquid-vapor contacting in distillation columns, data for plate design has been published by e.g. Stichlmair (1978), Perry (1984) and Lockett (1986). Key parameters as e.g. weeping, flooding and efficiency of sieve tray are influenced by the pressure drop which in turn is dependent on liquid and vapor flow as well as the tray design.

A detailed overview on recently published literature in the field of dynamics and control of distillation columns is given by Skogestad (1992). The survey includes the description of distillation models with rigorous and linearized tray hydraulics and a review of widely used simplifications. Rigorous models for distillation columns for nonlinear simulations are developed by e.g. Gani et al. (1986) and Retzbach (1986). The work of Gani et al. is focused on the development of a general dynamic model including tray hydraulics and accurate prediction of the physical properties which is numerically robust. Further the influence of the simplifying assumption was investigated. The rigorous model of Retzbach is primarily developed for the nonlinear simulation of a multi-component mixture in a distillation column with side stripper. The tray hydraulic models applied by Retzbach are extensively described by Stichlmair (1978).

The paper is divided into 5 parts. We present a detailed description of the rigorous stage model in Section 2. The liquid holdup is divided into liquid on the sieve tray and downcomer. The dynamic model is implemented in the SPEEDUP simulation environment (1992) with a link to the ASPEN PROPERTIES PLUS data base for thermodynamic properties (1988). The third part deals with linearized tray hydraulics which simplify the dynamic model considerably. In the fourth section we present different methods to determine hydraulic parameters which describe the dynamic behavior of the system. These methods are based on experiments on our lab scale column or developed based on the rigorous stage model. Finally the results of the different methods are compared and conclusions are presented.

\section{Tray modeling}

Tray models based on first principle consist of a large number of differential algebraic equations which may be solved simultaneously. Simulation of a staged distillation where the dynamics is described from first principles will enable a thorough investigation of the tray hydraulics. Changes in column design will influence the distribution of liquid on the stages and change the time constants of the system. A deeper insight in the hydraulics of a stage will reduce the possibility of designing a distillation column which is inherently difficult to control.

\subsection{Rigorous tray model}

In the rigorous model implemented in the SPEEDUP simulations each stage is divided into two liquid holdups (tray and downcomer) and one vapor holdup. Separated mass and energy balances for tray and downcomer are set up. The holdups are computed from the mass balances, the flows leaving the tray are determined from 


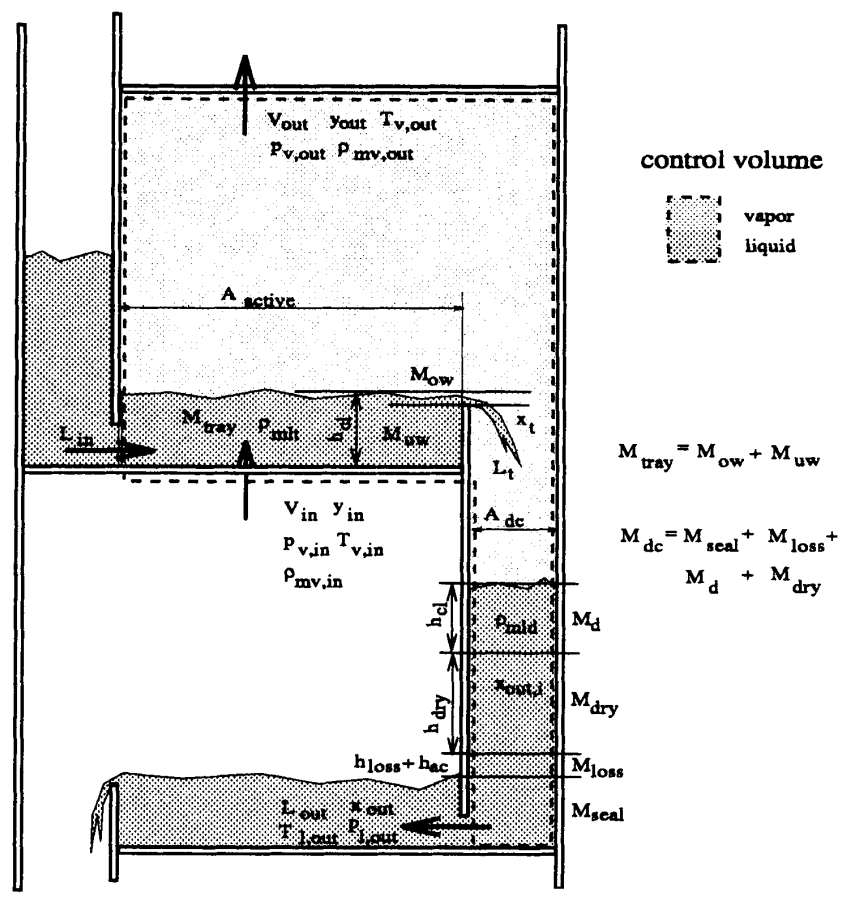

Figure 1. Typical design of a staged distillation column with sieve tray and downcomer.

hydraulic correlations with pressure drop over the stage as driving force. The thermodynamic properties of the components are calculated by the ASPEN PROPERTIES PLUS (1988) package. The main difficulty in these simulations was to find the steadystate operating points which match the performed experiments.

The design of a typical stage in a distillation column consisting of sieve tray, downcomer, inlet and outlet weir is shown in Figure 1.

Assumptions. The total holdup of the tray consists of liquid and vapor holdup. According to the geometry of the interior of the distillation column, see Figure 1, the liquid holdup on a stage is divided into liquid on the active tray area and liquid in the downcomer. The following assumptions are made:

R1 two-phase system in thermal and mechanical equilibrium

R2 perfect mixing in vapor and liquid phases

R3 no heat losses to the surroundings

R4 no heat of mixing

R5 temperature dynamics of the column structure is neglected

Conservation of internal energy and constant volume of the system implies that the flash calculation is solved as an UV-flash.

Material and energy balance. The balances presented are used throughout the whole distillation model, some modifications are made for the reboiler and accumulator. The mathematical states are the component holdup $M$ (both in vapor and liquid phase) on the stage and the internal energy. An in depth overview of these equations is given in Lockett (1983) and Stichlmair (1978). 
The following set of equations is valid for a tray without feed, side draw and external heating or cooling.

$$
\frac{d M_{i}}{d t}=V_{i n} \cdot y_{i n, i}+L_{i n} \cdot x_{i n, i}-V_{\text {out }} \cdot y_{\text {out }, i}-L_{t} \cdot x_{t, i}
$$

The holdup of component $i$ on the tray, this is liquid on the tray and vapor above tray and downcomer is

$$
M_{i}=M_{t} \cdot x_{t, i}+M_{v} \cdot y_{\text {out }, i}
$$

The total molar holdup on the tray is given by

$$
\sum_{i=1}^{n} M=M_{t}+M_{v}
$$

The energy balance of the tray

$$
\frac{d U_{t}}{d t}=V_{\text {in }} \cdot h_{v, \text { in }}+L_{\text {in }} \cdot h_{l, \text { in }}-V_{\text {out }} \cdot h_{v, \text { out }}-L_{t} \cdot h_{l, t}
$$

where the total internal energy is described by

$$
U_{t}=M_{v} \cdot\left(h_{v, \text { out }}-\frac{p}{\rho_{v}}\right)+M_{l} \cdot\left(h_{l, \text { out }}-\frac{p}{\rho_{l}}\right)
$$

A similar set of equations is applied to the downcomer material balance:

$$
\begin{gathered}
\frac{M_{d c, i}}{d t}=L_{t} \cdot x_{t, i}-L_{\text {out }, i} \cdot x_{\text {out }, i} \\
M_{d}=\sum_{i=1}^{n} M_{d c, i}
\end{gathered}
$$

the component balance:

$$
M_{d c, i}=M_{d} \cdot x_{o u t}
$$

the energy balance of the downcomer

$$
\frac{U_{d c}}{d t}=L_{t} \cdot h_{l, t}-L_{\text {out }} \cdot h_{l, o u t}
$$

and the total internal energy of the downcomer

$$
U_{d c}=M_{d} \cdot h_{l, o u t}
$$

where we neglect the pressure dependent part. Note that there is no flash calculation performed on the downcomer holdup. The vapor composition is in equilibrium with the liquid on the tray and the vapor volumes of the tray and downcomer are combined. Due to this assumption a vapor flow from the downcomer to the tray can be neglected such that the overall system is somewhat simplified. Due to the assumption that the pressure is identical, both in the liquid phase of the tray and downcomer as well as the vapor phase the computation of the molar density is simplified.

Holdup distribution. The liquid and vapor molar holdup a stage is related to the total tray volume $V_{t}$ by: 


$$
V_{t}=M_{t} / \rho_{l}+M_{v} / \rho_{v}+M_{d c} / \rho_{l}
$$

The molar volume of the liquid on the tray $M_{t}$ and in the downcomer $M_{d c}$ is computed by

$$
M_{t}=h_{c l} \cdot A_{\text {active }} \cdot \rho_{l, t}
$$

and

$$
\left.M_{d c}=M_{c l}+M_{d r y}+M_{\text {loss }}+M_{\text {seal }}=(\underbrace{\left(h_{c l}+h_{d r y}+h_{\text {loss }}\right.}_{h_{d c}}) \cdot A_{d c}+V_{\text {seal }}\right) \cdot \rho_{l, d}
$$

where $V_{\text {seal }}$ is the volume of the downcomer seal. We assume no bubbles in the downcomer so $h_{d c}$ is the height of clear liquid. The pressure drop over the downcomer (Perry et al., 1984) from the surface of the liquid exiting the downcomer to the surface of the downcomer level is identical to the total pressure drop, $\Delta p$, over a plate (see equation (20)). The height $h_{d r y}$ is computed from the 'dry' pressure drop over the holes of the tray (see equation (23)). The flow under the downcomer apron is modeled by a nonstationary Bernoulli equation which considers losses due to friction $\left(h_{\text {loss }}\right)$ and acceleration of the liquid under the downcomer apron (see Figure 1).

Vapor-liquid equilibrium. The thermodynamic equilibrium of vapor and liquid is defined by

$$
y_{\text {eq }, i}=K_{i} \cdot x_{\text {out }, i}
$$

with a K-value dependent on $x, y, T, p$. The composition of the vapor leaving the tray is computed by the Murphree tray efficiency coefficient

$$
\eta_{i}=\frac{y_{\text {out }, i}-y_{\text {in }, i}}{y_{\text {eq }, i}-y_{\text {in }, i}}
$$

with $y_{e q, i}$ as the equilibrium vapor composition at given tray composition, temperature and pressure. It is assumed that liquid and vapor are perfectly mixed in their control volumina.

\subsection{Tray hydraulics}

The modeling of the tray hydraulic is based on empirical correlations selected from the literature. Lockett (1986) and Stichlmair (1978) give an excellent overview over the different approaches. The chosen correlations are not necessarily the best available, but allow fairly accurate predictions and are easy to implement.

Clear liquid height. Consider the stage shown in Figure 1. Recall equation (12) where the liquid holdup on the sieve tray, $M_{t}$, is defined with the active tray area, $A_{t}$, (excluding downcomer) and the clear liquid height $h_{c l}$. The clear liquid height is less than the actual height of fluid on the plate due to bubbles dispersed in the liquid. The fraction of liquid (froth density) in the fluid is denoted $\phi$.

$$
h_{c l}=\phi h_{w e i r}+h_{o w}
$$

We use the correlation presented by Bennett (1983) to compute the froth density $\phi$

$$
\phi=\exp \left(\pi_{1} \cdot K_{s}^{\pi_{2}}\right)
$$


with the superficial velocity factor

$$
K_{s}=v_{t} \sqrt{\rho_{v} /\left(\rho_{l}-\rho_{v}\right)}
$$

where $v_{t}=V_{i n} /\left(A_{t} \rho_{v}\right)$ is the vapor velocity over the active tray. The parameters $\pi_{1}$ and $\pi_{2}$ are empirical constants. Bennett uses $\pi_{1}=-12.55$ (in units consistent with the velocity factor $K_{\mathrm{s}}$ in $\mathrm{m} / \mathrm{s}$ ), and $\pi_{2}=0.91$ (dimensionless). We fitted new values to match pressure drop experiments and to get reasonable values for the liquid holdup.

Flow over outlet weir. The liquid flow, $L_{\text {out }}$, over the circular weir from the tray to the downcomer, is computed with a modified Francis weir formula (Perry et al., 1984). Since the outlet weir is placed off center towards the column wall, the liquid height above the weir $h_{o w}$ will not be constant. There are no existing correlations which deal with the converging flow over circular outlet weirs in distillation column (Lockett, 1986). Taking the design into consideration, we choose to correct the weir length with a factor of $0 \cdot 5$.

$$
h_{\text {ow }}=44300 \cdot\left(\frac{L_{\text {out }}}{\rho_{l} \cdot 0 \cdot 5 \cdot d_{\text {weir }}}\right)^{0.704}
$$

We have here assumed that the liquid contains no bubbles as it passes over the weir, that is, $h_{o w}$ is the clear liquid height of liquid over the weir (see equation (16)).

The actual height of fluid (mixture of vapor bubbles and liquid) on the tray is then $h_{c l} / \phi$. If the computed froth height, $h_{c l} / \phi \leqslant h_{\text {weir }}$, the liquid flow leaving the tray is set to zero.

Pressure drop correlations. The pressure drop over a stage is measured as the difference in pressure between two adjacent stages

$$
\Delta p=p_{i}-p_{i-1}
$$

The total pressure drop $\Delta p$ consists of the 'static' (wet) pressure drop through the aerated liquid on the sieve tray and the 'dry' pressure drop through the holes of the tray, $\Delta p_{d r y}$.

$$
\Delta p=\Delta p_{\text {static }}+\Delta p_{d r y}
$$

The 'wet' pressure drop due to the height of clear liquid on the tray is

$$
\Delta p_{\text {static }}=g \cdot \rho_{l} \cdot h_{c l}
$$

According to Lockett (1986) the hydraulic gradient for sieve tray distillation column of small diameter (less than $0.5 \mathrm{~m}$ ) is negligible.

Numerous correlations for the dry pressure drop are available (e.g. Liebson et al., 1957) we have chosen:

$$
\Delta p_{d r y}=\rho_{m l} \cdot \frac{g}{1000} \cdot \frac{51}{C_{o}^{2}} \frac{\rho_{m v}}{\rho_{m l}} \cdot v_{h}^{2}=0 \cdot 965 \cdot \rho_{v} \cdot v_{h}^{2}
$$

where $v_{h}=v_{t} A_{t} / A_{h}$ is the velocity through the holes [m/s].

The pressure loss due to surface generation, $\Delta p_{\text {bubble }}$, is neglected compared to the equations presented in Coulson et al. (1983) or Perry et al. (1984). The 'residual pressure drop', $\Delta p_{\text {resid }}$, as listed in literature (e.g. Perry et al. (1984)) is neglected, since the suggested liquid height of $12.5 \mathrm{~mm}$ is comparable to the static liquid head on the sieve tray. 
Summary of holdup distribution. The total liquid holdup on a stage is

$$
M=M_{t}+M_{d c}
$$

Here $M_{t}$ is given by equations (12)-(19). We have

$$
M_{t}=\underbrace{A_{t} \rho_{l} \phi h_{w e i r}}_{M_{u w}}+\underbrace{A_{t} \rho_{l} h_{o w}}_{M_{o w}}
$$

Note that $\phi$ depends on the vapor flow $V$ whereas $h_{o w}$ depends on the liquid flow $L$. We identify four contributions to the liquid holdup in the downcomer.

$$
M_{d c}=M_{l o s s}+M_{c l}+M_{d r y}+M_{\text {seal }}
$$

1. Holdup which corresponds to the height of clear liquid on the tray plus the contribution from the corresponding "wet" pressure drop through the liquid on the tray above:

$$
M_{c l}=A_{d} \rho_{l} 2 h_{c l}=2 \frac{A_{d}}{A_{t}} M_{t}
$$

2. Holdup corresponding to pressure drop over the trays perforation ("dry pressure drop"), which from equation (23) is

$$
M_{d r y}=\frac{A_{d} \cdot \Delta p_{d r y}}{g \cdot M W_{l}}
$$

3. Holdup due to pressure drop under the downcomer apron, $M_{\text {loss }}$. This term may usually be neglected.

4. Holdup of liquid in the downcomer seal, $M_{\text {seal }}$, which is independent of vapor and liquid flow.

\section{Linear tray hydraulics}

In order to obtain further insight into the tray hydraulics we shall consider the linearized approximation. Such a simple approximation may also be used in order to simplify dynamic simulations. For simplicity, the vapor holdup is neglected and constant molar flows are assumed.

It is assumed that the liquid flow, $L_{\text {out }}$, is a function of the liquid holdup, $M$ and the vapor flow $V_{\text {in }}$ (Rademaker et al., 1975; Skogestad et al., 1988). Taking the total differential of $L_{\text {out }}$ then yields

$$
d L_{\text {out }}=\underbrace{\left(\frac{\delta L_{\text {out }}}{\delta V_{\text {in }}}\right)_{M}}_{\lambda} d V_{\text {in }}+\underbrace{\left(\frac{\delta L_{\text {out }}}{\delta M}\right)_{V}}_{1 / \tau_{L}} d M
$$

Note that this relationship is assumed to hold dynamically. The hydraulic time constant $\tau_{L}$ typically varies from 0.5 to 15 seconds. The vapor constant $\lambda$, representing the influence of $V_{\text {in }}$ on $L_{\text {out }}$, typically ranges between -5 to +5 (Rademaker et al., 1975).

We want to obtain $\lambda$ and $\tau_{L}$ from correlations for the liquid holdup $M$ as a function of liquid and vapor flow given in equations (24) to (28). The value of $\tau_{L}$ can be directly 
obtained from these correlations. To find $\lambda$ we note that the total differential of $M$ can be written

$$
d M=\underbrace{\left(\frac{\delta M}{\delta L_{\text {out }}}\right)_{V}}_{\tau_{L}} d L_{\text {out }}+\underbrace{\left(\frac{\delta M}{\delta V_{\text {in }}}\right)_{L}}_{\tau_{V}} d V_{\text {in }}
$$

(this equation holds only at steady state). $\tau_{L}$ is always positive whereas $\tau_{V}$ may be either positive or negative.

Setting $d M=0$ in equations (29) and (30) yields

$$
\lambda=\left(\delta L_{\text {out }} / \delta V_{\text {in }}\right)_{M}=-\left(\tau_{V} / \tau_{L}\right)
$$

Since both $\tau$ and $\lambda$ depend on the tray loading, their values may be significantly different for the rectifying and the stripping section.

To derive analytic expressions for $\tau_{L}, \tau_{V}$ and $\lambda$ the holdup expressions in equations (24) and (28) are linearized:

$$
\begin{gathered}
\frac{\delta M_{t}}{\delta V_{\text {in }}}=A_{t} \rho_{l} \pi_{2} \frac{\phi \ln \phi}{V_{\text {in }}} h_{\text {weir }}=\frac{M_{u w}}{V_{\text {in }}} \pi_{2} \ln \phi \\
\frac{\delta M_{\text {dry }}}{\delta V_{\text {in }}}=2 \frac{M_{\text {dry }}}{V_{\text {in }}} \\
\frac{\delta M_{\text {loss }}}{\delta L_{\text {out }}}=2 \frac{M_{\text {loss }}}{L_{\text {out }}} \\
\frac{\delta M_{\text {ow }}}{\delta L_{\text {out }}}=0.704 \frac{M_{\text {ow }}}{L_{\text {out }}}
\end{gathered}
$$

Equations (32) to (35) yield:

$$
\begin{gathered}
\tau_{V}=2 \frac{M_{\text {dry }}}{V_{\text {in }}}+\left(1+2 \frac{A_{d}}{A_{t}}\right) \frac{M_{u w}}{V_{\text {in }}} \pi_{2} \ln \phi \\
\tau_{L}=2 \frac{M_{\text {loss }}}{L_{\text {out }}}+\left(1+2 \frac{A_{d}}{A_{t}}\right) 0 \cdot 704 \frac{M_{\text {ow }}}{L_{\text {out }}}
\end{gathered}
$$

Note that for our trays which have downcomers which exit above the liquid, we replace $2\left(A_{d} / A_{t}\right)$ by $A_{d} / A_{t}$. It is important to note that $\tau_{V}$ and thus $\lambda$ may be either positive or negative. A negative $\lambda$ means that more liquid is stored inside the column when vapor flow is increased, resulting in a temporary reduction in liquid flow. The main cause for this effect is the increased pressure drop which increases $M_{d r y}$. A positive $\lambda$ means that less liquid is stored inside the column when vapor flow is increased. At first this may seem unlikely. However, in tray columns it is quite common and is caused by a 'swelling' (decrease in the froth density $\phi$ ) which pushes liquid off the trays. For situations where $\lambda \geqslant 0$ an increase in vapor flow rate results in a decrease of liquid holdup on the tray. The liquid which is displaced on the sieve tray will be dumped on the stage below. If the change in 'dry' pressure drop is extensive enough (increase in $h_{d r v}$ ) this excess liquid will probably be stored in the downcomer. 
Table 1. Numerical example, estimated data for experiments 5 and 6

\begin{tabular}{|c|c|c|c|c|c|}
\hline \multirow[b]{3}{*}{ Parameter } & \multirow[b]{3}{*}{ Dimension } & \multicolumn{2}{|c|}{ Experiment 5} & \multicolumn{2}{|c|}{ Experiment 6} \\
\hline & & Top & Bottom & Top & Bottom \\
\hline & & \multicolumn{4}{|c|}{ Section } \\
\hline$\Delta p_{d r y}(23)$ & $\mathrm{Pa} /$ tray & \multicolumn{2}{|c|}{199} & \multicolumn{2}{|c|}{247} \\
\hline$\phi(17)$ & & $0 \cdot 20$ & $0 \cdot 47$ & $0 \cdot 17$ & $0 \cdot 45$ \\
\hline$h_{o w}(19)$ & $\mathrm{mm}$ & $1 \cdot 43$ & $2 \cdot 16$ & $1 \cdot 43$ & $2 \cdot 16$ \\
\hline$M_{o w}(25)$ & mol & $0 \cdot 16$ & $0 \cdot 24$ & $0 \cdot 16$ & $0 \cdot 24$ \\
\hline$M_{u w}(25)$ & mol & 0.67 & $1 \cdot 60$ & $0 \cdot 59$ & $1 \cdot 52$ \\
\hline$M_{d r y}(28)$ & mol & \multicolumn{2}{|c|}{$0 \cdot 38$} & \multicolumn{2}{|c|}{$0 \cdot 47$} \\
\hline$\delta M / \delta V(32)$ & $\mathrm{s}$ & $-6 \cdot 27$ & $-5 \cdot 75$ & $-5 \cdot 33$ & $-5 \cdot 23$ \\
\hline$\delta M_{d r y} / \delta V(33)$ & $\mathrm{s}$ & \multicolumn{2}{|c|}{$5 \cdot 40$} & \multicolumn{2}{|c|}{$6 \cdot 02$} \\
\hline$\delta M_{o w} / \delta L(35)$ & $\mathrm{s}$ & $1 \cdot 32$ & $1 \cdot 11$ & $1 \cdot 32$ & $1 \cdot 11$ \\
\hline$\tau_{L}(37)$ & $\mathrm{s}$ & $1 \cdot 32$ & $1 \cdot 12$ & $1 \cdot 32$ & $1 \cdot 12$ \\
\hline$\tau_{V}(36)$ & $\mathrm{s}$ & -0.87 & $-0 \cdot 34$ & $0 \cdot 69$ & 0.79 \\
\hline$\lambda(31)$ & & 0.65 & $0 \cdot 31$ & $-0 \cdot 52$ & $-0 \cdot 70$ \\
\hline
\end{tabular}

Numerical example. We will discuss the experimental data later, but to illustrate the above procedure we shall compute analytically the holdup distribution and hydraulic parameters for the top and bottom section for experiments 5 and 6. Experiment 5 has a large liquid load and experiment 6 a large vapor load.

The key data for the stage design are the weir height, $h_{w e i r}=30 \mathrm{~mm}$, and the ratio of downcomer to active tray area $A_{d} / A_{t}=0 \cdot 123$. With these data and the coefficients for equation (17) presented in Table 2 we compute the data presented in Table 1 . We have chosen to estimate the coefficients for equation (17) for the rectifier and stripper section separately, since these sections have a rather different liquid load.

The column is operated with a feed of ethanol/butanol of composition $z_{F}=0.5$ and a feed flow of $350 \mathrm{ml} / \mathrm{min}$. The reboiler heat input is $Q_{B}=5 \cdot 79 \mathrm{~kW} \Rightarrow V=0 \cdot 142 \mathrm{~mol} / \mathrm{s}$ and $Q_{B}=6.45 \mathrm{~kW} \Rightarrow \mathrm{V}=0 \cdot 158 \mathrm{~mol} / \mathrm{s}$ for experiments 5 and 6 , respectively. The reflux is in both cases $L_{T}=470 \mathrm{ml} / \mathrm{min}=0.096 \mathrm{~mol} / \mathrm{s}$. Note we assume constant molar flows through the column.

The term $\delta M_{\text {loss }} / \delta L$ is of the order of $0.006 s$ and can be neglected compared to the other terms.

These two experiments differ in the reboiler effect by approximately $10 \%$, but give entirely different results when the hydraulic parameters are computed. Note that the two contributions to $\tau_{V}, \delta M_{d r y} / \delta V$ (contribution from change in downcomer holdup due to pressure drop) and $\delta M_{t} / \delta V$ (contribution from change in tray holdup due to froth density), change in different directions when the heat input to the reboiler is increased. It is then clear that $\tau_{V}$ may easily change sign, and so may then $\lambda=$ $-\tau_{V} / T_{L}$. For experiment 5 the absolute values of $\delta M_{d r y} / \delta V \leqslant \delta M_{t} / \delta V$ are such that the liquid replaced on the sieve tray can not be stored in the downcomer. For experiment 6 the situation is such that the replaced liquid will be stored in the downcomer.

In conclusion, the hydraulic parameter $\lambda$, representing the initial effect of vapor flow on liquid flow, may be very sensitive to operating contributions.

\subsection{Linear flow relationships for column sections}

In the following assume that the hydraulic parameters are equal for all trays in the top section $\left(\tau_{L T}\right.$ and $\left.\lambda_{T}\right)$, and for all trays in the bottom section $\left(\tau_{L B}\right.$ and $\left.\lambda_{B}\right)$. Consider 
a small deviation from steady state. With constant molar flows the material balance on a tray becomes

$$
\frac{d M}{d t}=d L_{\text {in }}-d L_{\text {out }}
$$

where the liquid flow leaving the tray is given from the linearized tray hydraulics (equation (29))

$$
d L_{\text {out }}=\lambda d V+\frac{1}{\tau_{L}} d M
$$

Repeated combination of these equations yields the following expression for the change in liquid flow at the bottom of the column, $L_{B}$, in response to changes in reflux, $L_{T}$ and boilup, $V$ : (similar to Rademaker et al., 1975; Skogestad et al., 1988):

$$
d L_{B}=g_{L}(s) d L_{T}+g_{V}(s) d V
$$

where

$$
\begin{aligned}
& g_{L}(s)=\frac{1}{\left(1+\tau_{L T} s\right)^{N_{T}}} \frac{1}{\left(1+\tau_{L B} S\right)^{N_{B}}} \\
& g_{V}(s)=\lambda_{B}\left(1-\frac{1}{\left(\tau_{L B} s+1\right)^{N_{B}}}\right)+\frac{\lambda_{T}}{\left(\tau_{L B}{ }^{s}+1\right)^{N_{B}}}\left(1-\frac{1}{\left.\tau_{L T} s+1\right)^{N_{T}}}\right)
\end{aligned}
$$

The response of $L_{B}$ to a change in reflux at the top, $L_{T}$ is as expected a cascade of first-order responses, one for each tray. The response of $L_{B}$ to a change in vapor flow, $V$, requires a more detailed derivation. Consider first a column with only one tray. We consider here the effect of a change in $V$ only, so set $d L_{i n}=0$ in equation (38). Taking Laplace transforms of equations (38) and (39) and combining yields

$$
\frac{1}{\tau_{L}} d M=-\frac{\lambda}{\tau_{L} s+1} d V
$$

and we get with one tray

$$
d L_{\text {out }}=d L_{1}=\lambda\left(1-\frac{1}{\tau_{L} s+1}\right) d V
$$

For two identical trays we get in addition the change in liquid flow from the tray above, $d L_{\text {in }}$, which will be equal to $d L_{1}$ given above. $d L_{\text {in }}$ will affect $d L_{\text {out }}$ through a first order lag, so the total effect of a change in vapor flow with two trays is

$$
d L_{\text {out }}=d L_{1}+\frac{d L_{1}}{\tau_{L} s+1}=\lambda\left(1-\frac{1}{\left.\tau_{L} s+1\right)^{2}}\right) d V
$$

For a column with $N$ identical trays we find

$$
d L_{\text {out }}=d L_{B}=\lambda\left(1-\frac{1}{\left(\tau_{L} S+1\right)^{N}}\right) d V
$$

For a column with a top and bottom section

$$
d L_{B}=\lambda_{B}\left(1-\frac{1}{\left(\tau_{L B} S+1\right)^{N_{B}}}\right) d V+\frac{d L_{T, \text { out }}}{\left(\tau_{L B} S+1\right)^{N_{B}}}
$$


where $L_{\text {out }, T}$ is the liquid entering from the top section. It is itself caused by the increase in vapor, so

$$
d L_{T, \text { out }}=\lambda_{T}\left(1-\frac{1}{\left(\tau_{L T} s+1\right)^{N_{T}}}\right) d V
$$

and we derive the desired expression for $g_{V}(s)$.

Introduce the following approximations (which are good for long column sections)

$$
\frac{1}{\left(1+\tau_{L B} s\right)^{N_{B}}} \approx e^{-\theta_{B} s} ; \quad \frac{1}{\left(1+\tau_{L T} s\right)^{N_{T}}} \approx e^{-\theta_{T} s}
$$

where $\theta_{B}=N_{B} \tau_{L B}$ and $\theta_{T}=N_{T} \tau_{L T}$. Also introduce the total liquid lag from the top to the bottom $\theta=\theta_{B}+\theta_{T}$. Then we get from equation (46) with $d L_{T}=0$

$$
d L_{B} \approx \lambda_{B}\left(1-\mathbf{e}^{-\theta_{B} s}\right) d V+\lambda_{T}\left(\mathbf{e}^{-\theta_{B} s}-\mathbf{e}^{-\theta_{s}}\right) d V
$$

In words, a step increase in boilup, $d V$, will result in an immediate increase in $L_{B}$ equal to $\lambda_{B} d V$. This increase will last approximately for the time $\theta_{B}$ (which is the time it takes for a change in liquid flow to propagate through the bottom section), then the increase in $L_{B}$ will change to the value $\lambda_{T} d V$ and it will stay at this value for an additional time $\theta_{T}$. After approximately time $\theta=\theta_{B}+\theta_{T}$ the liquid flow $L_{B}$ will change back to its original value.

\section{Obtaining parameters from experiments}

\subsection{Liquid holdup}

The liquid holdup on the stages determine the composition dynamics of the distillation column and influence the hydraulics of the system. The liquid holdup can be estimated from experiments and theoretical calculations.

(i) Experimental dumping. The column is emptied by turning of the reflux and the heating. Liquid evaporated during this operation is collected in the accumulator. The combined holdup change of accumulator and reboiler is then the amount of liquid stored on the trays in the column during normal operation.

(ii) Theoretical tray calculations using geometric data and correlations, see equations (24) to (28).

(iii) Tracer experiments. One may inject a heavy component at the top of the column and measure the time it takes for the wave to move down the column. In general, the time constant for the composition response on an individual tray is $\tau_{c}=M_{i} /(L+K V)$, where we can set $K=0$ for a heavy tracer, and the time constant (apparent delay) for $N$ trays in series is approximately $N \tau_{c}$. We approximate $\theta_{c} \simeq N \tau_{c}$ such that we are able to estimate the column holdup from the measured delay $\theta_{c}$.

(iv) Experimental temperature responses. Measuring the initial temperature response to a step change in reflux $\Delta L$ or vapor flow $\Delta V$ enables the estimation of the liquid holdup.

The necessary assumptions are:

OP1 vapor holdup is negligible

OP2 constant molar liquid holdup $M$ 
OP3 equimolar flows (simplified energy balance)

OP4 the local slope of the vapor-liquid equilibrium curve is $K_{i}$

OP5 local linear relation between liquid composition and temperature, $x_{i}=k \cdot T_{i}$.

The material balance over a stage becomes

$$
M d x_{i} / d t=L_{i}\left(x_{i-1}-x_{i}\right)+V_{i}\left(y_{i+1}-y_{i}\right)
$$

For a step change in $L_{i}$ and $V_{i}$ the internal flows are $L_{i}=L_{i}^{o}+\Delta L$ and $V_{i}=V_{i}^{o}+\Delta V$. Subtract the steady state solution from equation (50) and consider the time immediately after the step change when the tray composition still is unchanged. This yields the following relationship for the initial slope as a function of $\Delta L$ and $\Delta V$ :

$$
M\left(\frac{d \Delta x_{i}}{d t}\right)_{\text {init }}=\Delta L_{i}\left(x_{i-1}^{o}-x_{i}^{o}\right)+\Delta V_{i}\left(y_{i+1}^{o}-y_{i}^{o}\right)
$$

Apply assumptions OP4 and OP5 and rearrange equation (51)

$$
M_{i}\left(\frac{d \Delta T_{i}}{d t}\right)_{\text {init }}=\Delta L_{i}\left(T_{i-1}^{o}-T_{i}^{o}\right)+\Delta V_{i} K_{i}\left(T_{i+1}^{o}-T_{i}^{o}\right)
$$

From equation (52) it is possible to estimate the molar holdup $M_{i}$ on a stage from observing the initial slope of the tray temperature to changes in reflux or boilup.

\subsection{Liquid hydraulic time constant $\tau_{l}$}

The liquid hydraulic time constant can be determined experimentally by making a change in reflux and observing the delay in liquid response through the column.

(i) This delay may be observed from the response of the uncontrolled reboiler level, or

(ii) from the temperature responses inside the column.

(iii) $\tau_{L}$ may also be estimated theoretically from tray data (see equation (37)).

\subsection{Vapor constant $\lambda$}

The vapor constant $\lambda$ represents the initial effect of a change in vapor flow on liquid flow from a stage. Experimentally we can obtain $\lambda$ by the following means.

(i) From temperature responses. For example, for $\lambda \geqslant 1$ the effect of an increase in $V$ will initially be counteracted by an even larger increase in $L$ and we will observe an increase in tray temperature in the upper part of the column earlier than in the lower part.

(ii) From reboiler level response. Since $d M_{B} / d t=L_{B}-V$ we get from equation (49) that for a unit step in vapor flow $V$ (keeping the reflux flow constant $\Delta L=0$ ), the slope of the response of $M_{B}$ as a function of time will be approximately $\left(\lambda_{B}-1\right)$ for the time $\theta_{B}$, then equal to $\left(\lambda_{T}-1\right)$ for the next time $\theta_{T}$, and then remain at -1 .

(iii) Estimate $\lambda$ theoretically using hydraulic relationships such as equation (36) and equation (37).

\section{Results}

The distillation column is equipped with 11 sieve trays (numbered from the top) of $125 \mathrm{~mm}$ diameter. As compared with what could be expected in an industrial 
column, the hole area of the trays is relatively small $\left(A_{h}\right.$ is about $5 \%$ of $\left.A_{t}\right)$, and the holdup in the downcomer is relatively large (about $50 \%$ of total holdup). Also, the downcomer design is different from that in Figure 1 in that the downcomer ends above the liquid surface and has a quite large downcomer seal holdup.

The reboiler is a thermosyphon reboiler with a nominal holdup of 3.5 liters and equipped with electrical heating of a maximum effect of $15 \mathrm{~kW}$. The total condenser is connected to an accumulator with a holdup of approximately 1.5 liters. The reflux and feed are fed to the column by metering pumps and enter the column with a temperature approximately $2^{\circ} \mathrm{C}$ below the boiling point.

Each tray is equipped with a thermocouple which is placed approximately $10 \mathrm{~mm}$ above the center of the tray. Below the lowest tray a pressure sensor is installed to measure the pressure drop over the column. The distillation column is interfaced to a computer system for data recording and control with a sampling frequency of $1 \mathrm{~Hz}$.

The experiments were performed with mixtures of ethanol and butanol. For the theoretical estimation of holdups and time constants we assume constant density $\rho_{l}=731 \cdot 5 \mathrm{~kg} / \mathrm{m}^{3}$ (Halles, 1976) and mole weight $M W_{l}=60 \cdot 1 \mathrm{~kg} / \mathrm{kmol}$ corresponding to a $50 / 50$ mixture. We also assume $\Delta h_{\text {vap }}=40900 \mathrm{~kJ} / \mathrm{kmol}$ (Majer, 1985).

\subsection{Pressure drop}

Experiments were performed to measure the pressure drop over the distillation column. Based on these measurements and applying equations (16) to (23) we compute by means of a least square approach the coefficients which were used in equation (17). The estimation is performed separately for stripping and rectifier section. A comparison between experimental data and the estimated pressure drop is shown in Figure 2.

The empirical parameters $\pi_{1}$ and $\pi_{2}$ for equation (17) determined from the pressure drop measurements are presented in Table 2.

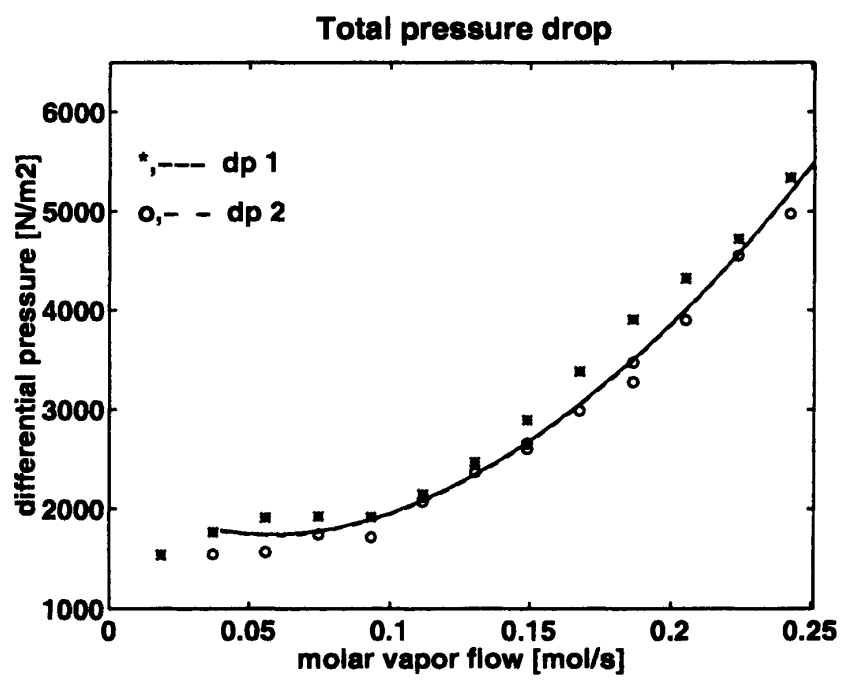

Figure 2. Comparison of measured $(*, o)$ and estimated $(-)$ pressure drop over the distillation. 
Table 2. Empirical parameters for equation (17)

\begin{tabular}{lcc}
\hline & $\pi_{1}$ & $\pi_{2}$ \\
\hline rectifier section & $-24 \cdot 17$ & 0.73 \\
stripping section & -7.05 & 0.61
\end{tabular}

\subsection{Liquid holdup}

Table 3 lists experimental conditions and some results for experiments 2, 3, 5 and 6.

(i) The liquid volume determined by dumping the distillation column is denoted $V_{l, \text { dump }}$. The corresponding average tray holdup is denoted $M_{l, \text { dump }}$.

(ii) The liquid volume estimated from pressure drop measurements is denoted $V_{l, \Delta p}$. It is found by measuring the differential pressure drop over the column and estimating the liquid holdup based on equations (24) to (28).

(iii) We performed experiments with only ethanol in the column and used butanol as a heavy tracer. With reflux $L=472 \mathrm{ml} / \mathrm{min}$ the time for the butanol to reach the bottom was about 330 seconds (see Figure 3), corresponding to a liquid holdup of approximately 2.591 .

The agreement between these three methods is good and gives a total column holdup of approximately $2 \cdot 7$ liters.

(iv) We also used equation (52) to estimate the holdup from the initial temperature response on trays 3 and 9 to a step response in heat input experiment 2 and experiment 6 as well as in reflux experiment 3 and experiment 5 . The temperature responses are shown in Figures 4 and 5 for experiments 2 and 3, respectively. The corresponding estimated holdups (denoted $M_{3}$ and $M_{9}$ )

Table 3. Data for holdup estimation for experiments 2, 3, 5 and 6

\begin{tabular}{|c|c|c|c|c|c|}
\hline & Units & Experiment 2 & Experiment 3 & Experiment 5 & Experiment 6 \\
\hline$F$ & $\mathrm{ml} / \mathrm{min}$ & 250 & 250 & 350 & 350 \\
\hline$z_{F}$ & & $0 \cdot 54$ & 0.56 & $0 \cdot 56$ & $0 \cdot 45$ \\
\hline$Q_{t=0}$ & $\mathrm{~kJ} / \mathrm{s}$ & 3.60 & $4 \cdot 50$ & $5 \cdot 70$ & $6 \cdot 45$ \\
\hline$\Delta Q$ & $\mathrm{~kJ} / \mathrm{s}$ & +0.45 & $0 \cdot 0$ & $0 \cdot 0$ & +1.58 \\
\hline$L_{t=0}$ & $\mathrm{ml} / \mathrm{min}$ & $249 \cdot 8$ & 382 & 468 & 470 \\
\hline$\Delta L$ & $\mathrm{ml} / \mathrm{min}$ & $0 \cdot 0$ & $+52 \cdot 8$ & $+88 \cdot 4$ & $0 \cdot 0$ \\
\hline$\Delta p$ & mbar & $22 \cdot 3$ & $26 \cdot 1$ & $35 \cdot 3$ & $54 \cdot 6$ \\
\hline$V_{l}$, dump & 1 & $2 \cdot 68$ & $2 \cdot 71$ & $2 \cdot 93$ & $3 \cdot 10$ \\
\hline$V_{l},{ }_{\Delta p}$ & 1 & $2 \cdot 77$ & $2 \cdot 74$ & $2 \cdot 69$ & $2 \cdot 66$ \\
\hline$M_{l}$, dump & mol & $3 \cdot 06$ & $2 \cdot 99$ & $3 \cdot 24$ & $3 \cdot 43$ \\
\hline$T_{2}$ & ${ }^{\circ} \mathrm{C}$ & $79 \cdot 12$ & $89 \cdot 91$ & $79 \cdot 17$ & $79 \cdot 74$ \\
\hline$T_{3}$ & ${ }^{\circ} \mathrm{C}$ & $79 \cdot 45$ & $84 \cdot 64$ & $79 \cdot 78$ & $81 \cdot 45$ \\
\hline$T_{4}$ & ${ }^{\circ} \mathrm{C}$ & $80 \cdot 42$ & $89 \cdot 91$ & $81 \cdot 09$ & $84 \cdot 65$ \\
\hline$d \Delta T_{3} / d t$ & $10^{-2}{ }^{\circ} \mathrm{C} / \mathrm{s}$ & $+0 \cdot 18$ & -1.43 & -0.63 & $+4 \cdot 23$ \\
\hline$M_{3}$ & mol & $6 \cdot 02$ & $3 \cdot 70$ & $2 \cdot 25$ & $3 \cdot 19$ \\
\hline$T_{8}$ & ${ }^{\circ} \mathrm{C}$ & $96 \cdot 72$ & $109 \cdot 71$ & $110 \cdot 73$ & $115 \cdot 64$ \\
\hline$T_{9}$ & ${ }^{\circ} \mathrm{C}$ & $104 \cdot 53$ & $113 \cdot 65$ & $114 \cdot 96$ & $116 \cdot 75$ \\
\hline$T_{10}$ & ${ }^{\circ} \mathrm{C}$ & $111 \cdot 08$ & $115 \cdot 78$ & $116 \cdot 61$ & $117 \cdot 21$ \\
\hline$d \Delta T_{0} / d t$ & $10^{-2 \mathrm{o}} \mathrm{C} / \mathrm{s}$ & $+4 \cdot 51$ & -0.87 & $-2 \cdot 23$ & $+2 \cdot 15$ \\
\hline$M_{9}$ & mol & $4 \cdot 14$ & $6 \cdot 39$ & $4 \cdot 46$ & $3 \cdot 37$ \\
\hline
\end{tabular}




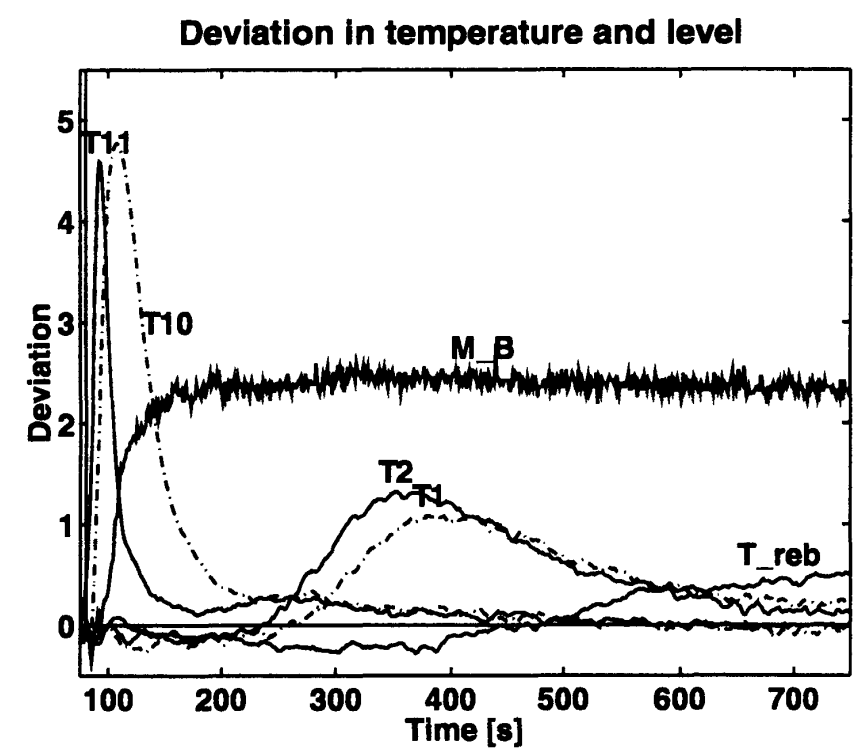

Figure 3. Deviation in temperature of reboiler, $T_{r e b}$ and on stages $1,10,11$ and the reboiler level response due to injection of a heavy tracer.

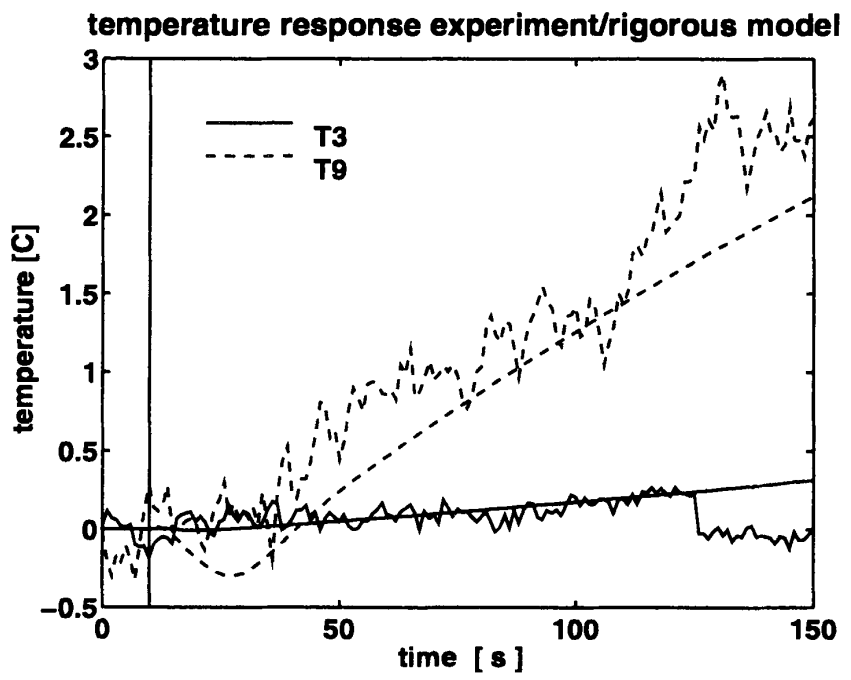

Figure 4. Experiment 2. Initial response of temperatures on trays 3 and 9 to a step change in heat input at $\mathrm{t}=10 \mathrm{~s}$.

show large variations (see Table 3 ) and are also different (mostly larger) from the values found above using dumping. The reason for the variations may be inaccuracy in determining the initial temperature derivative (see e.g. Figure 5). However, the deviation from the dumping experiments may also be because the assumptions for deriving equation (52) do not hold. This is partly confirmed by the simulated responses (smooth lines in the Figures) which are in good 


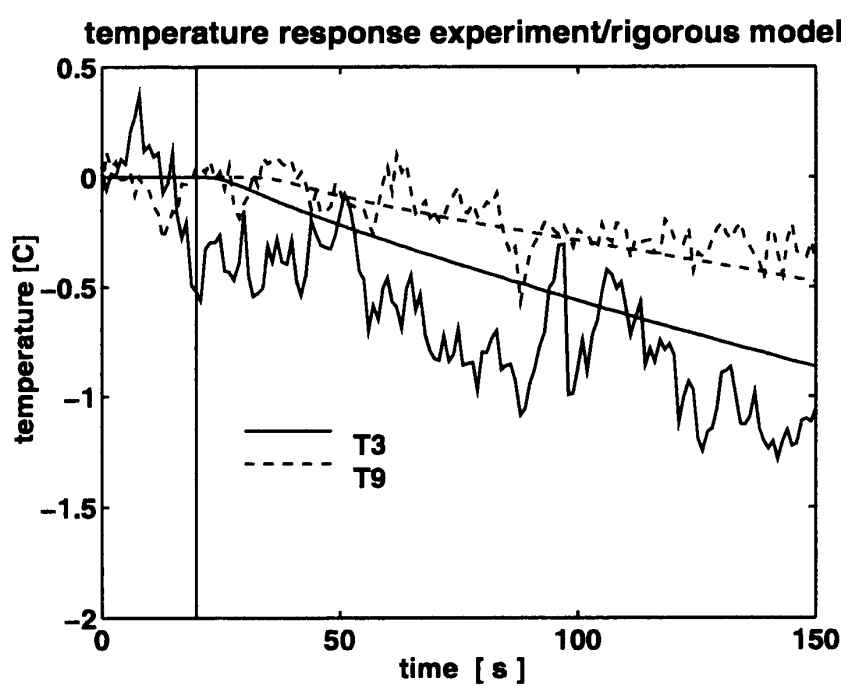

Figure 5. Experiment 3. Initial response of temperatures on trays 3 and 9 to a step change in reflux flow at $\mathrm{t}=20 \mathrm{~s}$.

agreement with the experiments. The simulated responses are for the full model (with no assumption about constant molar flow etc.) using holdups which are in agreement with the dumping experiments.

Compare experiments 2 to 6 and experiments 3 to 5 shows that the quality of prediction increase for increasing step changes. The changes in experiments 5 and 6 are at least twice the step changes as in experiments 2 and 3. Further we see that the estimated holdup varies largely for experiments 2 and 3. Inspecting the results show that the holdup is very much overpredicted for the opposite side of the column where the step was introduced.

\subsection{Liquid hydraulic time constant $\tau_{l}$}

For the experimental determination of the hydraulic time constant, the level control of the inventory is placed into manual, such that the positions of the product valves are fixed. The reboiler level will now primarily depend on the liquid flow to the reboiler and the vapor leaving it, although it should be noted that a fixed valve position not necessarily means that the liquid flow through the valve is constant.

Let us first consider experiment 5 .

(i) The hydraulic lag between the increase in reflux until the reboiler level changes is illustrated in Figure 6 . We find $\theta=23.1 \mathrm{~s}$ from the change in reflux pump control signal to the initial level change of the reboiler, which gives an average hydraulic time constant of $\tau_{L T}=2 \cdot 1 \mathrm{~s}$. The experimental response show a delay between the change in control signal and the change in accumulator level of $5 \cdot 3 \mathrm{~s}$. Consider the time difference from the initial accumulator level deviation until the reboiler changes which give a time delay of $\Delta \theta_{l}=17.8 \mathrm{~s}$. From this delay we compute an average hydraulic time constant for each stage of $\tau_{L}=1.62 \mathrm{~s}$. 


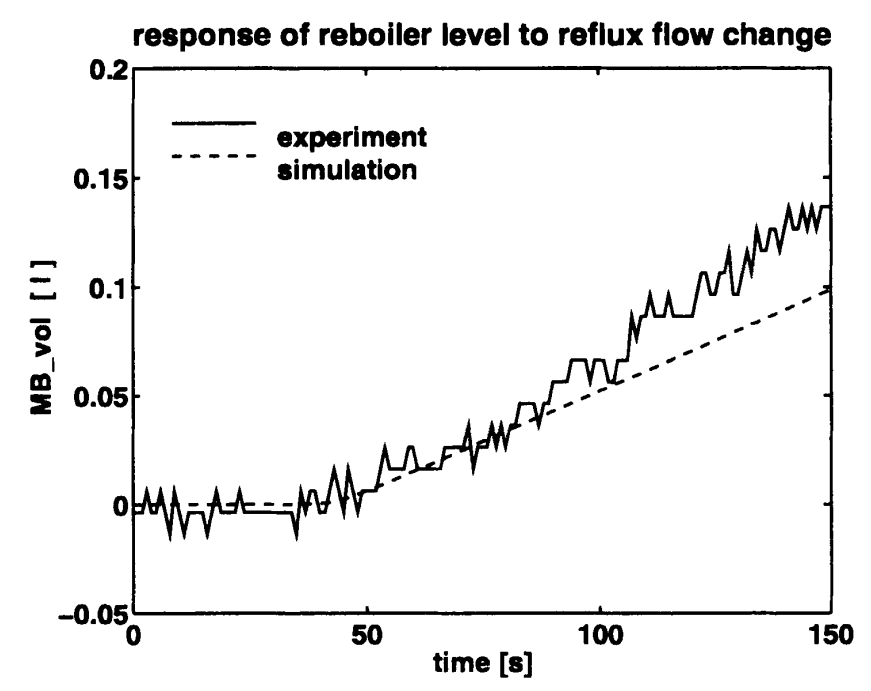

Figure 6. Experiment 5. Response of reboiler level to a step in reflux flow at $\mathrm{t}=20 \mathrm{~s}$.

(ii) Using temperature measurements we find that the liquid delay between the temperature change on trays 3 and 9 is 12.7 seconds, which give an average hydraulic time constant of $\tau_{L}=1 \cdot 68 \mathrm{~s}$. For experiment 3 we find a delay in the temperature change between the trays of 11.8 seconds which yields a hydraulic time constant of $1.68 \mathrm{~s}$.

(iii) Applying equation (37) to the set of operational conditions of experiment 5 given in Table 4 yields $\tau_{L T}=1.32 \mathrm{~s}$ and $\tau_{L B}=1.11 \mathrm{~s}$, which is approximately $2 / 3$ of the experimental value. For experiment 3 we find from the temperature responses in Figure 5 an average hydraulic time constant of $1.83 \mathrm{~s}$ the estimated time constant is 1.46 and 1.23 seconds for the rectifier and stripper, respectively.

We note from these and other results that the agreement between experiment and tray calculations is somewhat better for the level measurements if we consider the dynamics of the pump. Considering the dynamics in pump and sensor (see $(i)$ ) by

Table 4. Experiments 3 and 5 for changes in liquid flows. Experimental conditions, measured time delays $(\theta)$, and hydraulic time constant $\left(\tau_{L}\right)$

\begin{tabular}{lccr}
\hline & Units & Experiment 3 & Experiment 5 \\
\hline$F$ & $\mathrm{ml} / \mathrm{min}$ & 250 & 350 \\
$z_{F}$ & & $0 \cdot 56$ & $0 \cdot 56$ \\
$L_{t=0}$ & $\mathrm{ml} / \mathrm{min}$ & $329 \cdot 2$ & $468 \cdot 8$ \\
$L_{t=\infty}$ & $\mathrm{ml} / \mathrm{min}$ & $382 \cdot 7$ & $557 \cdot 2$ \\
$Q_{t=0}$ & $\mathrm{~kJ} / \mathrm{s}$ & $4 \cdot 50$ & $5 \cdot 8$ \\
$\Theta_{T 3}$ & $\mathrm{~S}$ & $16 \cdot 7$ & $13 \cdot 3$ \\
$\Theta_{T 9}$ & $\mathrm{~S}$ & $28 \cdot 5$ & 26 \\
$\Theta_{D}$ & $\mathrm{~S}$ & $*$ & $5 \cdot 3$ \\
$\Theta_{B}$ & $\mathrm{~S}$ & $*$ & $23 \cdot 1$ \\
$\tau_{L, t}$ (Eq. 37) & $\mathrm{S}$ & $1 \cdot 46$ & $1 \cdot 32$ \\
$\tau_{L, b}$ (Eq. 37) & $\mathrm{S}$ & $1 \cdot 23$ & $1 \cdot 11$ \\
\hline
\end{tabular}




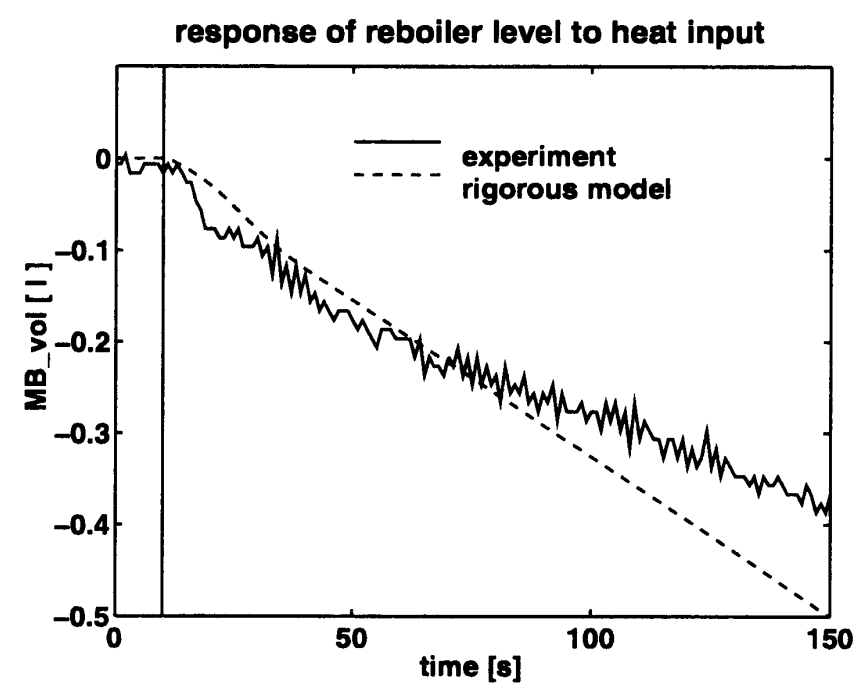

Figure 7. Experiment 6. Response of reboiler level to a step in heat input at $\mathrm{t}=10 \mathrm{~s}$.

redefining the time horizon reduce the deviation between the different methods considerably. The computed value from the temperature measurement (ii) is strongly dependent on the quality of the signal to get a good approximation of the hydraulic time constant.

Still, it seems that the estimated values for $\tau_{L}$ from equation (37) are too small. However, the rigorous simulations which are based on the same correlations show better agreement as is illustrated by comparing the experimental and simulated reboiler level in Figure 6. The reason for the difference may be a somewhat more detailed tray model and effects of the energy balance which are also not included in the simple linear analysis leading to equation (37).

\subsection{Vapor constant $\lambda$}

$\lambda$ may be observed from the initial response to changes in the boilup as changes in the slope of the tray temperature or reboiler level as a function of time. From the temperature measurements for experiment 2 it is difficult to observe any change in the slope so one would expect $\lambda$ to be close to 0 . This does not agree with estimated values from tray calculations which yield $\lambda$ equal to $5 \cdot 1$ and $3 \cdot 8$ in the top and bottom sections, respectively. As seen from the simulated temperature on tray 9 , this should yield an inverse response, which should be easy to observe in the bottom section (dashed curve in Figure 4).

From the level measurement for experiment 6 one can from Figure 7 observe a change in the slope after about $10 \mathrm{~s}$ and then another change after about $35 \mathrm{~s}$ (from when the change in heat input was applied). This indicates that $\theta_{T}$ is about $25 \mathrm{~s}$ and $\theta_{B}$ is about $10 \mathrm{~s}$. This is in reasonable agreement with experiment 5 which has the same initial liquid flow (see Table 1). If we consider the slope of $M_{B}$ as a function of time then one would from the experimental response in Figure 4 expect $\lambda_{T}$ to be about -1 and $\lambda_{B}$ to be about -3 .

Again, this does not agree very well with the estimated values of about -0.52 and $-0 \cdot 70$, even if the sign $\lambda_{T}$ and $\lambda_{B}$ is correct. This deviation may seen very large, but 
the estimated values are very sensitive to small changes in the data. For example, consider experiment 6 for which we estimate $\lambda_{B}=-0 \cdot 7$. Recall from equation (36) that $\lambda$ has two contributions with different signs (see Table 1). Both of these are very sensitive to the value of the vapor flow. For example, the magnitude of the pressure drop term (which yields a negative contribution to $\lambda$ ) is proportional to $V$, while the froth density term (which yields a positive contribution to $\lambda$ ) may become less important as $V$ is increased (because the froth density $\phi$ cannot be less than 0 ). For experiment 6, we obtained in Section $3 \tau_{V B}=6.02 \mathrm{~s}-5.23 \mathrm{~s}=-0.70 \mathrm{~s}$.

If we decrease the vapor flow by $20 \%$ then we find $\tau_{V B}=4.812 \mathrm{~s}-6.32 \mathrm{~s}=-1.51 \mathrm{~s}$. $\tau_{L}$ is unchanged at $1 \cdot 11 \mathrm{~s}$, so we find that a $20 \%$ decrease in $V$ changes the estimated value of $\lambda_{B}$ from -0.70 to 1.35 such that an inverse response for the bottom section can be expected. On the other hand, an increase in vapor flow by $20 \%$ changes the estimated $\lambda_{B}$ from $-0 \cdot 70$ to $-2 \cdot 49$, which is rather close to the experimental value of about -3 .

\section{Discussion and conclusion}

The agreement with the experimental responses and simulations using the detailed model were generally very good, except for the initial effect of changes in vapor flow (e.g. as expressed by the parameter $\lambda$ ). However, this was obtained only after considerable effort (including some parameter fitting) so in general we believe it is difficult to predict accurately the initial response of distillation columns based on only geometric data about the column and thermodynamic data. Two reasons are:

1. The correlations for estimating the tray holdups are empirical and do not seem very reliable. For example, this applies to the formula for estimating the froth density.

2. Even with a good model for estimating the tray holdups, the dynamic response, for example as expressed by $\lambda$, is very sensitive to small changes in the parameters. This means that one cannot expect to be able to predict from tray data alone whether one will have an inverse response to changes in boilup.

In general, the inverse response is undesirable so one would like to have $\lambda<0 \cdot 5$. Fortunately, the models give us insight into how the tray design should be changed to achieve this. The reason for the undesired positive contribution to $\lambda$ is the swelling due to bubbles. This effect seems difficult to avoid and it is probably desirable to improve mass transfer. To make $\lambda$ more negative one needs to increase the downcomer holdup as vapor flow is increased. This may be achieved by using a larger downcomer area or by increasing the pressure drop over the trays (e.g. by using smaller holes in the plates). Another alternative, which may be more attractive, is to use a packed column where liquid holdup generally increases with vapor flow, implying that $\lambda$ is generally negative.

For control purposes one may want $\tau_{L}$ large as this increases the liquid lag from the top to the bottom of the column, and thus tends to decouple the dynamic response in the two column ends. To increase $\tau_{L}$ one may increase $M_{o w}$ by using a shorter outlet weir. Alternatively, one may increase the pressure drop under the downcomer to increase the term $M_{\text {loss }}$. For packed columns all the liquid contributes to the liquid lag, $\tau_{L}$ (as there is no holdup under the weir or in the downcomer), so although the liquid holdup in a packed column is generally smaller than in a trayed column, the decoupling effect from the liquid flow dynamics (as expressed by $\tau_{L}$ ) may not be any less. Thus packed columns are generally preferred compared to trayed columns when it comes to control considerations. 
In conclusion, the dynamic simulations based on a detailed model gave good agreement with experimental responses, while it was found that simpler models based on constant molar flows and linear tray hydraulics gave quite large deviations. Better correlations are needed for predicting the holdup on the trays for the rigorous model. However, even with improved correlations one cannot expect to get accurate predictions of the flow behavior (e.g. $\lambda$ ) because of strong sensitivity to parameter changes. One will therefore in most cases need experimental data to validate the responses and possibly adjust parameters in the model for the tray hydraulics.

\section{REFERENCES}

ASPEN PROPERTIES PLUS (1988). User Guide, Release 8, Aspen Technology, Inc., Cambridge, USA.

Baber, M. F., Edwards, L. L., Harper, W. T., Witte, M. D. and Gerster, J. A. (1961). Experimental Transient Response of a Pilot Plant Distillation Column, Process Dynamics and Control, 57(36), 149-159.

Baber, M. F. and Gerster, J. A. (1962). Experimental Transient Response of a Pilot Plant Distillation Column: Part II. Response to Liquid and Vapor Rate Perturbations, AIChE Journal, 8(3), 407-415.

Bennett, D. L., Agrawal, R. and Cook. P. J. (1983). New Pressure Drop Correlations for Sieve Tray Distillation Columns, AIChE Journal, 29(3), 434-443.

Coulson, J. M., Richardson, J. F. and Sinnott, R. K. (1985). Chemical Engineering, Volume 6, Design, Pergamon Press.

Gani, R., Ruiz, C. A. and Cameron, T. (1986). A generalized Model for Distillation Columns-I: Model Description and Application, Computers \& Chemical Engineering, 10(3), 181-198.

Hales, J. L. and Ellender, J. H. (1976). Liquid densities from 293 to $490 \mathrm{~K}$ of nine aliphatic alcohols, J. Chem. Thermodynamics, 8, 1177-1184.

Liebson, I., Kelly, R. E. and Bullington, L. A. (1957). How to design perforated trays, Petrol. ref., 36(127).

Lockett, M. J. (1986). Distillation tray fundamentals, Cambridge University Press.

Majer, V. and Svoboda, V. (1985). Enthalpies of Vaporization of Organic Compounds, Chemical Data Series, No. 32.

Perry, H. P. and Green, D. (1984). Perry's Chemical Engineers' Handbook, McGraw-Hill Chemical Engineering Series, 50th edition, Chapter 18.

Rademaker, O., Rijnsdorp, J. E. and MaArleveld, A. (1975). Dynamics and Control of Continuous Distillation Units, Elsevier, Amsterdam.

Retzbach, B. (1986). Mathematische Modell von Destillationskolonnen zur Synthese von Regelungskonzepten, VDI Fortschrittberichte, Reihe 8: Mess-, Steuerungs- und Regelungstechnik, Nr. 126.

Skogestad, S. and Morari, M. (1988). Understanding the Dynamic Behavior of Distillation Columns, Ind. \& Eng. Chem. Research, 27(10), 1848-1862.

Skogestad, S. (1992). Dynamics and Control of Distillation Columns-A critical Survey, IFACSymposium DYCORD +'92, Maryland.

SPEEDUP (1992). Release 5.3 User Manual, Prosys Technology Ltd.

STICHLMair, J. (1978). Grundlagen der Dimensionierung des Gas/Flüssig Kontaktapparates Bodenkolonne, Verlag Chemie, Weinheim, New York.

\section{Notation}

A area

$C_{0} \quad$ discharge coefficient

d diameter

$F_{i} \quad$ molar feed flow of component $i$

$g \quad$ standard acceleration of gravity

$h$ molar enthalpy

$h$ height $\mathrm{m}^{2}$

$\mathrm{mm}$

$\mathrm{kmol} / \mathrm{h}$

$\mathrm{m} / \mathrm{s}^{2}$

$\mathrm{GJ} / \mathrm{kmol}$

$\mathrm{mm}$ 


\begin{tabular}{|c|c|c|}
\hline$K$ & equilibrium constant & \\
\hline$k$ & constant & \\
\hline$K_{s}$ & dimensionless velocity & \\
\hline$l$ & characteristic length & $\mathrm{mm}$ \\
\hline$L_{i}$ & molar liquid flow of component $i$ & $\mathrm{kmol} / \mathrm{h}$ \\
\hline$M$ & molar holdup & $\mathrm{kmol}$ \\
\hline$M W$ & molecular weight & $\mathrm{kg} / \mathrm{kmol}$ \\
\hline$N$ & Number of trays & \\
\hline$p$ & pressure & bar, $N / m^{2}$ \\
\hline$q$ & liquid flow & $\mathrm{m}^{3} / \mathrm{s}$ \\
\hline$Q$ & heat input & $\mathrm{kJ} / \mathrm{s}$ \\
\hline $\bar{T}$ & temperature & ${ }^{\circ} \mathrm{C}$ \\
\hline$v$ & velocity & $\mathrm{m} / \mathrm{s}$ \\
\hline$V$ & molar vapor flow & $\mathrm{kmol} / \mathrm{h}$ \\
\hline Vol & volume & $\mathrm{m}^{3}$ \\
\hline$y_{e q, i}$ & equilibrium molar fraction vapor & \\
\hline$y_{i}$ & molar fraction of component $i$ & \\
\hline$x_{i}$ & molar fraction of component $i$ & \\
\hline$z_{i}$ & molar fraction of component $i$ & \\
\hline \multicolumn{3}{|c|}{ Greek Symbols } \\
\hline$\Delta p$ & pressure drop & $\mathrm{N} / \mathrm{m}^{2}$ \\
\hline$\Delta L$ & change in liquid flow & $\mathrm{kmol} / \mathrm{h}$ \\
\hline$\Delta Q$ & change in heat input & $\mathrm{kJ} / \mathrm{s}$ \\
\hline$\rho_{m}$ & liquid mass density & $\mathrm{kg} / \mathrm{m}^{3}$ \\
\hline$\rho$ & liquid molar density & $\mathrm{kmol} / \mathrm{m}^{3}$ \\
\hline$\phi$ & froth density & \\
\hline$\theta$ & time delay & $\mathrm{s}$ \\
\hline$\lambda$ & vapor constant & \\
\hline$\tau$ & time constant & $\mathrm{s}$ \\
\hline$\pi$ & empirical parameter & \\
\hline \multicolumn{3}{|c|}{ Subscripts } \\
\hline$a$ & downcomer apron area & \\
\hline cl & clear liquid & \\
\hline$d, d c$ & downcomer & \\
\hline dry & parameter reled to dry pressure drop & \\
\hline$e q$ & equilibrium composition & \\
\hline$h$ & hole area of the tray & \\
\hline$i$ & identifier & \\
\hline in & flow into the system volume & \\
\hline$n$ & index for number of components & \\
\hline$l$ & liquid phase & \\
\hline loss & hydrodynamic losses & \\
\hline out & flow out of the system volume & \\
\hline ow & over weir & \\
\hline seal & seal pan of downcomer & \\
\hline stage & stage consisting of tray and downcomer & \\
\hline$t$ & tray & \\
\hline tot & total & \\
\hline$u w$ & under weir & \\
\hline$v$ & vapor phase & \\
\hline weir & weir geometry & \\
\hline
\end{tabular}

